\title{
Effect of apparatus dead space on breathing parameters in newborns: "flow-through" versus conventional techniques
}

\author{
G. Schmalisch*, B. Foitzik*, R.R. Wauer*, J. Stocks\#
}

\begin{abstract}
Effect of apparatus dead space on breathing parameters in newborns: "flow-through" versus conventional techniques. G. Schmalisch, B. Foitzik, R.R. Wauer, J. Stocks. (C) ERS Journals Ltd 2001.

ABSTRACT: Commercial devices for tidal breathing measurements in newborns allow only short-term measurements, due to the high apparatus dead space of the face mask and pneumotachometer. The flow-through technique (FTT) minimizes the dead space by a background flow, thereby allowing long-term measurements. The aim of this study was to investigate the comparability of tidal breathing parameters using both techniques.

Paired measurements of tidal breathing were performed in 86 sleeping infants (median (range) body weight $2.8 \mathrm{~kg}(1.9-5.3 \mathrm{~kg})$, age 65 days (3-150 days)), using the FTT and SensorMedics 2600 (SM 2600).

There was a significant bias $(\mathbf{p}<\mathbf{0 . 0 0 1})$ in all tidal breathing parameters. Compared with the FTT, increases $(95 \%$ confidence interval $(C I))$ in tidal volume $(V T)$, respiratory frequency $(f \mathrm{R})$, and minute ventilation $\left(V^{\prime} \mathrm{E}\right)$ were $0.74(0.5-1.0) \mathrm{mL} \cdot \mathrm{kg}^{-1}, 9.0$ $(6.9-11.2) \cdot \mathrm{min}^{-1}$ and $92(74-109) \mathrm{mL} \cdot \mathrm{min}^{-1} \cdot \mathrm{kg}^{-1}$ when measured with the SM 2600 , representing average increases of 13,17 and $30 \%$, respectively, in response to the added dead space. By contrast, time to peak tidal expiratory flow as a proportion of expiratory time ( $t$ PTEF $/ t$ E) was changed by -0.09 (-0.11--0.08). The mean $(95 \% \mathrm{CI})$ change in $t$ PTEF $t$ tE of $-54(-62-45) \%$, when measured in infants by the SM 2600, was remarkably similar to that observed during in vitro validation studies $(-59(-73--44) \%)$, suggesting that the discrepancies in timing parameters may be largely attributable to differences in signal processing.

In conclusion, differences in measurement technique and precision of the devices used can result in significant differences in tidal breathing parameters. This may impede the comparison of results within and between infants and the clinical interpretation of tidal breathing measurements in newborns.
\end{abstract}

Eur Respir J 2001; 17: 108-114.

\author{
*Dept of Neonatology (Charité), Hum- \\ boldt University, Berlin, Germany, \\ ${ }^{\#}$ Portex Anaesthesia, Intensive Therapy \\ and Respiratory Medicine Unit, Insti- \\ tute of Child Health, London, UK. \\ Correspondence: G. Schmalisch, Dept \\ of Neonatology (Charité), Humboldt \\ University Berlin, Schumannstraße 20/ \\ 21, D-10098, Berlin, Germany. \\ Fax: 493028025824 \\ Keywords: Apparatus dead space \\ flow-through technique \\ infants \\ pneumotachography \\ tidal breathing \\ ventilation
}

Received: June 301999

Accepted after revision July 262000

This study was supported by the German Ministry for Research and Technology, project "Perinatal Lung"

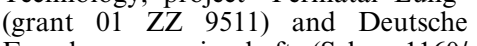
Forschungsgemeinschaft (Schm 1160/ $1-2)$.
Most methods for respiratory function testing (RFT) in newborns currently require an accurate measurement of ventilation and correct recognition of the beginning of each inspiration and expiration. With the exception of forced expiratory manoeuvres, ventilatory measurements in infants are most commonly performed during tidal breathing [1]. While both respiratory inductance [2] and "face out" body plethysmography [3] have been used to record tidal breathing without any facial attachments, thereby eliminating all external dead space, the former only provides quantitatively accurate measurements if measurement conditions remain very stable [4], while the latter is too expensive and cumbersome for routine bedside application [5]. Consequently, direct measurement of airflow at the airway opening using a pneumotachometer (PNT) or similar flow meter connected to a face mask, has remained the most popular option for quantitative measurements.

Unfortunately, while being very simple, this measurement technique influences the very parameters it is designed to measure, respiration being stimulated by irritation of the nucleus trigeminus during facial attachment of the mask [6,7], the additional resistance of the pneumotachometer (RPNT) and, most importantly, the apparatus dead space ( $V \mathrm{D}, \mathrm{app})[8]$. The latter is of particular concern in newborns due to their low absolute tidal volumes $\left(5-8 \mathrm{~mL} \cdot \mathrm{kg}^{-1}\right.$ body weight) [9]. While recent technological advances have facilitated the development of low dead space flowmeters, there are limitations to how much the effective dead space of the mask (which often represents the greatest component of $V \mathrm{D}$,app during tidal breathing measurements) can be reduced [10]. Since the water displacement of the smallest available face mask appears to be in the order of $10 \mathrm{~mL}$, about half of which will represent effective dead space once in situ, this will present a considerable challenge to spontaneously breathing newborns, especially those $<3 \mathrm{~kg}$. Consequently, the duration of RFTs in newborns is limited if the conventional technique is applied. However, representative recordings of tidal breathing often require a relatively long acquisition period due to the high variability of sleep state and respiratory signals, especially in preterm newborns. RUTTIMANN et al. [11] described a method for overcoming this problem in ventilated neonates, by using differential 
pneumotachography based on flow measurements in the inspiratory and expiratory limb of the respirator circuit. This technique was subsequently used for ventilatory measurements in intubated, spontaneously breathing infants [12]. An adaptation of this technique for tidal breathing measurements using a face mask is shown in figure 1 . This technique was called the flowthrough technique (FTT) [13] because the face mask and PNT are continuously and thoroughly rinsed by a constant background flow which virtually eliminates $V \mathrm{D}$,app. In contrast to the conventional technique, the functional reduction of $V \mathrm{D}$,app by FTT allows longterm tidal breathing measurements.

The aim of this study was to investigate the comparability of the most commonly measured tidal breathing parameters, using both techniques, in newborns.

\section{Materials and methods}

\section{Subjects}

Tidal breathing measurements were performed in 86 newborns (table 1) over a 10-month period (November 1996-August 1997), in the respiratory function laboratory of the Dept of Neonatology at Humboldt University (Charité, Berlin, Germany). The measurements were part of a prospective follow-up study of infants requiring mechanical ventilation during the neonatal period. In addition to other lung function tests such as the measurement of lung volume [14], paired measurements of tidal breathing parameters were performed using the conventional method and the flow-through technique. Admission criteria to this study were: spontaneous ventilation, quiet sleep according to PRECHTL [15], and written parental consent. Infants with major malformations, upper airway obstruction, acute respiratory infection, congenital heart disease or central

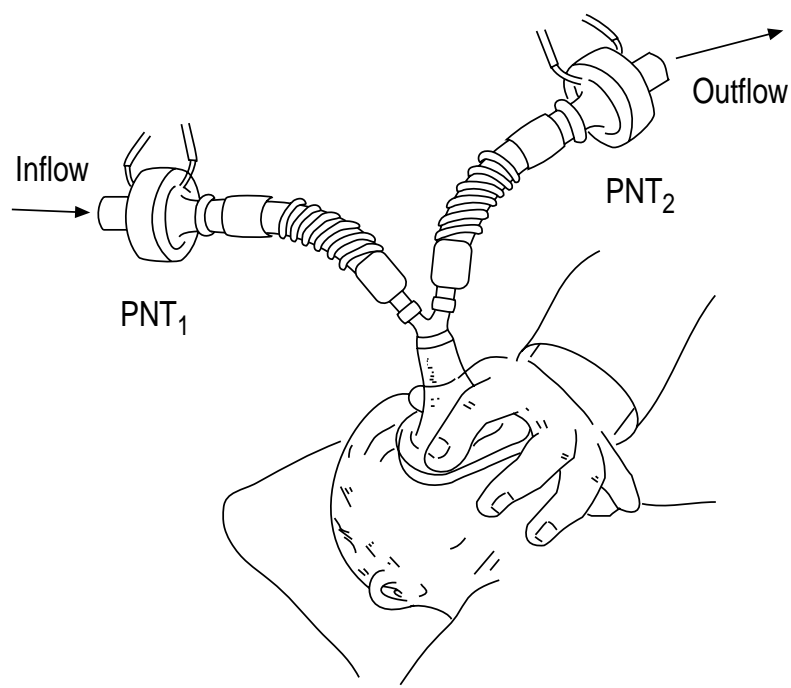

Fig. 1. - Tidal breathing measurements in newborns using the flow-through technique in which the face mask is continuously rinsed by a constant background flow and the infant's ventilation is given by the flow difference between the two pneumotachographs $\left(\mathrm{PNT}_{1} ; \mathrm{PNT}_{2}\right)$.
Table 1. - Patient characteristics

\begin{tabular}{lc}
\hline Parameter & Median (range) \\
\hline Body weight g & $2800(1880-5300)$ \\
Body length cm & $49(38-57)$ \\
Age days & $65(3-150)$ \\
Postconceptional age weeks & $40.4(34.0-49.4)$ \\
Sedation incidence & 27 out of $86 / 31 \%$ \\
\hline
\end{tabular}

nervous malfunction were not eligible for recruitment. This study was approved by the medical faculty (Charité) of the Humboldt University (protocol 54/ 92). Parents were given a full explanation of the tests and equipment used before their consent was obtained.

\section{Equipment for tidal breathing measurements}

For the conventional tidal breathing measurements, the SensorMedics 2600 (SM 2600; SensorMedics Inc., Anaheim, CA, USA) was used, as previously described [16]. Ventilatory measurements were performed using transparent face masks for premature neonates (size 1) or infants (size 2) (Vital Signs Inc., Totowa, NJ, USA), connected to a PNT (flow range: $0-10 \mathrm{~L} \cdot \mathrm{min}^{-1}$, $R P N T=0.5 \mathrm{kPa} \cdot \mathrm{L}^{-1} \cdot \mathrm{s}$ total resistance of the PNT defined by the back pressure at $5 \mathrm{~L} \cdot \mathrm{min}^{-1}$, Hans Rudolph Inc., Wyandotte, MI, USA). The $V \mathrm{D}$, app of the face mask and PNT was $9 \mathrm{~mL}$ for mask size 1 and $18 \mathrm{~mL}$ for mask size 2, determined by water displacement using a head model. Integration of airflow to derive volume was performed at $256 \mathrm{~Hz}$ without any filtering of the raw signals [16]. The calibration of the PNT, including the linearization procedure, was performed prior to each measurement, according to the recommendations of the manufacturer. Custom made equipment was used for the tidal breathing measurements using the FTT (fig. 1). The inflow and outflow of the face mask were measured by two screen PNTs (linear range $0-240 \mathrm{~L} \cdot \mathrm{min}^{-1}, R \mathrm{PNT}=0.2 \mathrm{kPa} \cdot \mathrm{L}^{-1} \cdot \mathrm{s}$, total resistance, defined by the back pressure at $5 \mathrm{~L} \cdot \mathrm{min}^{-1}$, Baby PNT Jaeger, Würzburg, Germany) and the infant's airflow was given by the difference between the two flow signals. The continuous background flow, which was generated by an air mixer and flow regulator, was fed into the inspiratory limb and the same face mask as used with the SensorMedics 2600 (SM 2600) via a modified y-piece. Flow was recorded at $200 \mathrm{~Hz}$ and filtered by an analogue Bessel filter of fourth order with a cut-off frequency of $48 \mathrm{~Hz}$ to avoid aliasing [17]. The software Labview (Version 4.0.1., National Instruments, Austin, Texas, USA) was used for data acquisition and further signal processing. The in vitro measurement accuracy of the two devices was assessed as described previously, using a mechanical model pump to generate flow profiles that simulated those observed in infants [18].

\section{Measurements in infants}

Normally, a clean, dry newborn falls asleep in comfortable environmental conditions within about 
half an hour of feeding. This biological reaction and the infant's sleep-waking rhythm were used to determine timing of respiratory function tests. Most infants were studied during natural, quiet sleep, assessed by behavioural criteria [15], but 27 children $(31 \%)$ were sedated with chloral hydrate $\left(50 \mathrm{mg} \cdot \mathrm{kg}^{-1}\right)$ given orally $15-30 \mathrm{~min}$ before testing. Sedation was primarily necessary in older infants and for the additional lung function tests, such as measurement of lung volume. Parents were usually present during the RFT. For the measurements, sleeping infants were placed in a supine position with the neck in a neutral position, supported by a head seal. After the infant was asleep, tidal breathing measurements were performed, firstly with the FTT, followed immediately afterwards with the SM 2600. The duration of both measurements was normally $20-30 \mathrm{~min}$, depending on the period of acclimatization to the face mask. Infants were continuously monitored throughout the whole procedure by pulse oximetry.

During the FTT, the constant background flow ( $V^{\prime}$ const) was adjusted in such a way that it was always higher than the infant's peak tidal inspiratory flow [13]. After a sufficient period of adaptation (5-20 min), tidal breathing was measured and the airtight seal of the mask on the infant's face was checked by continuous leak monitoring [19]. The end of the adaptation period is commonly characterized by a more regular breathing pattern without any visible drift in breathing parameters. When using the FTT, on-line display of time based signals and flow/volume loops facilitate both recognition of the end of the period of adaptation and selection of suitable epochs, with low breath-to-breath variability and minimal artefacts for subsequent evaluation. The graphical display of instantaneous respiratory frequency over the last 60 breathing cycles also assists assessment of the stability of the tidal breathing parameters.

Depending on the variability of the breathing pattern, at least 10 , but not more than 30 consecutive breaths were evaluated. Immediately afterwards, tidal breathing measurements were carried out with the SM 2600. An identically sized face mask connected to the Rudolph PNT was placed on the face. When using the SM 2600, single breathing cycles of the stored signals have to be selected by the investigator. For the current study, tidal breathing parameters were determined from 4-12 selected breathing cycles when the infant was breathing regularly.

\section{Statistical methods}

Mean \pm SD was calculated for all ventilatory parameters, together with the mean $(95 \%$ confidence interval (CI)) of the within-subject differences in tidal breathing parameters between the two methods. The comparability of the two techniques was assessed using paired t-tests and the statistical approach described by BLAND and Altman [20]. The software STATGRAPHICS (Version 4.0, Manugistics Inc., Rockville, Maryland, USA) was used for the statistical evaluations. A level of statistical significance of $p<0.05$ was accepted.

\section{Results}

The comparison of the tidal breathing parameters recorded with both methods are shown in table 2, where statistically significant differences between techniques for all parameters investigated $(\mathrm{p}<0.001)$ are displayed. Thus, when compared with the FTT, tidal volume $(V \mathrm{~T})$, respiratory frequency $(f \mathrm{R})$ and minute ventilation $\left(V^{\prime} \mathrm{E}\right)$ were on average 13,17 and $30 \%$ higher, respectively, when measured by the SM 2600. Even greater differences were found for parameters dependent on the shape of the flow signal or the tidal breathing flow/volume loop (TBFVL), with mean \pm SD time to peak tidal expiratory flow as a portion of expiratory time ( $t \mathrm{PTEF} / t \mathrm{E})$ decreasing from $0.24 \pm 0.08$ (FTT) to $0.15 \pm 0.08$ (SM 2600), representing a mean $(95 \% \mathrm{CI})$ change in $t \mathrm{PTEF} / \mathrm{tE}$ of $-54(-62--45) \%$. The parameter volume to peak tidal expiratory flow as a portion of tidal volume ( $V \mathrm{PTEF} / V \mathrm{~T})$, which describes the site of maximum expiratory flow on the TBFVL, and which is strongly correlated with $t \mathrm{PTEF} / \mathrm{tE}(\mathrm{r}=$ 0.959 for SM 2600 and $r=0.834$ for FTT), was also found to have decreased (by about 39\%) during measurements with the SM 2600. By contrast, the

Table 2. - Comparison of tidal breathing parameters measured in infants with SensorMedics 2600 (SM 2600) and flowthrough technique (FTT)

\begin{tabular}{|c|c|c|c|c|}
\hline \multirow{2}{*}{ Parameter } & \multicolumn{2}{|c|}{ Group means } & \multicolumn{2}{|c|}{ Mean individual differences $(95 \% \mathrm{CI})(\mathrm{SM} 2600-\mathrm{FTT}){ }^{* * *}$} \\
\hline & SM 2600 & FTT & Absolute difference & Relative difference $\%$ \\
\hline$V \mathrm{~T} \mathrm{~mL} \cdot \mathrm{kg}^{-1}$ & $6.1 \pm 1.5$ & $5.3 \pm 1.4$ & $0.74(0.5-1.0)$ & $12.9(8.0-17.8)$ \\
\hline$f_{\mathrm{R}} \min ^{-1}$ & $58.6 \pm 13.0$ & $49.5 \pm 11.8$ & $9.0(6.9-11.2)$ & $16.8(12.8-20.9)$ \\
\hline$V^{\prime} \mathrm{E} \mathrm{mL} \cdot \mathrm{min}^{-1} \cdot \mathrm{kg}^{-1}$ & $349 \pm 97$ & $258 \pm 74$ & $92(74-109)$ & $29.5(24.5-34.5)$ \\
\hline$t \mathrm{E} \mathrm{s}$ & $0.61 \pm 0.16$ & $0.74 \pm 0.19$ & $-0.12(-0.16--0.09)$ & $-18.0(-22.5-13.4)$ \\
\hline$t \mathrm{PTEF} / t \mathrm{E}$ & $0.15 \pm 0.08$ & $0.24 \pm 0.08$ & $-0.09(-0.11--0.08)$ & $-53.5(-62.4-44.6)$ \\
\hline$V \mathrm{PTEF} / V \mathrm{~T}$ & $0.20 \pm 0.09$ & $0.29 \pm 0.07$ & $-0.085(-0.1--0.07)$ & $-39.4(-46.7--32.0)$ \\
\hline TEF25 L $\cdot \mathrm{min}^{-1} \cdot \mathrm{kg}^{-1}$ & $0.56 \pm 0.20$ & $0.44 \pm 0.14$ & $0.12(0.09-0.16)$ & $23.2(16.9-29.6)$ \\
\hline
\end{tabular}

Group means are presented as mean $\pm \mathrm{SD}$, within-subject differences between measurement techniques are shown as absolute and relative mean difference with the $95 \%$ confidence interval $(\mathrm{CI}): V \mathrm{~T}$ : tidal volume; $f \mathrm{R}$ : respiratory frequency; $V^{\prime} \mathrm{E}$ : minute ventilation; $t \mathrm{E}$ : expiratory time; $t \mathrm{PTEF} / \mathrm{tE}$ : time to peak tidal expiratory flow as a proportion of expiratory time; $V \mathrm{PTEF} / V \mathrm{~T}$ : volume to peak tidal expiratory flow as a portion of tidal volume; TEF25: expiratory flow when $25 \%$ of tidal volume remains in the lung. $* * *$ : for all $\mathrm{p}<0.001$ for difference between SM 2600 and FTT. 

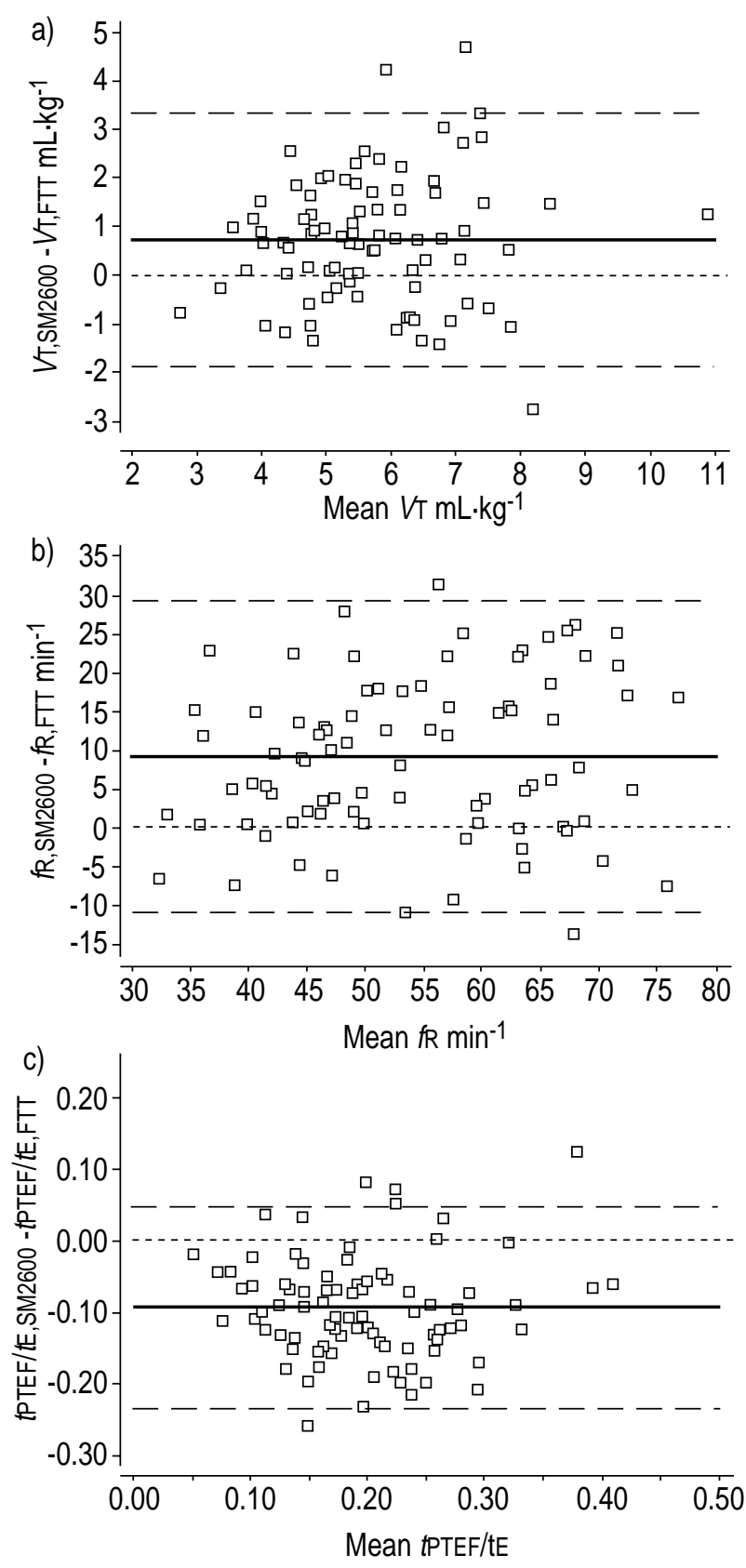

Fig. 2. - Scatter plots, according to the method of BLAND and Altman [20], of differences in tidal volume $(V \mathrm{~T})$, respiratory frequency $(f \mathrm{R})$ and time to peak tidal expiratory flow as a proportion of tidal expiratory time $(t \mathrm{PTEF} / t \mathrm{E})$ between the SM 2600 and flow-through technique (FTT). The mean within-subject difference (-) and the $95 \%$ limits of agreement (- -; $1.96 \mathrm{SD})$ are indicated. Mean within subject differences for $V \mathrm{~T}, f \mathrm{R}$ and $t \mathrm{PTEF} /$ $t \mathrm{E}$ are $0.7 \mathrm{~mL} \cdot \mathrm{kg}^{-1}, 9.0 \cdot \mathrm{min}^{-1}$ and -0.09 , respectively.

parameter expiratory flow when $25 \%$ of tidal volume remains in the lung (TEF25), which reflects the curvature of the end-expiratory limb of the TBFVL, was significantly higher $(\mathrm{p}<0.001)$ when measured with the SM 2600. Individual within-subject differences for $V \mathrm{~T}, f \mathrm{R}$ and $t \mathrm{PTEF} / t \mathrm{E}$ are shown in figure 2 , plotted according to the BLAND and ALTMAN method [20]. It can be seen that while there was considerable individual variability to the change in measurement technique, with wide limits of agreement between the methods, a significant bias was evident in all the measured parameters (table 2).

\section{Discussion}

\section{Relative advantages and limitations of the two methods}

The conventional technique, which simply requires a face mask and low-dead space PNT, is widely used for RFT in newborns. The physical properties of the gas flow through the PNT (room air during inspiration, alveolar gas with body temperature and pressure saturated (BTPS) conditions during expiration) can be corrected [21]. In contrast to the FTT, it can be used in conjunction with techniques requiring airway occlusions (e.g. plethysmography and passive respiratory mechanics [1]). However, a crucial disadvantage of this method is the high apparatus dead space, which in newborns often exceeds the physiological dead space. The duration of measurements, therefore, needs to be as short as possible in such infants to avoid compromising alveolar gas exchange. This disadvantage can be overcome by using the FTT. The virtual elimination of $V \mathrm{D}$,app by the background flow permits long-term measurements, the duration of which can readily be adapted to the prevailing measuring conditions (e.g. time required to reach a steady state) as well as to check the variability of the respiratory signals. In addition, the FTT has the advantage that the inspired oxygen fraction can be adjusted, tracer gases for other RFTs can be added [13], and it can easily be used in infants requiring continuous positive airway pressure [22]. Furthermore, the FTT can be combined with oesophageal manometry [23] or forced oscillations to investigate lung mechanics during tidal breathing [24]. The FTT also allows continuous assessment of air leaks between the face and the mask which is helpful for maintaining airtight placement of the face mask [19].

Potential disadvantages of the FTT include the higher technical expenditure and demands on both PNTs (e.g. linear characteristics required over a wider flow range to avoid any influence of the background flow on the measurement). In addition, as mentioned previously, the FTT cannot be used for all respiratory function tests, particularly those requiring airway occlusions. Nevertheless, it would appear to be the method of choice for tidal breathing measurements in newborns, especially those delivered prematurely.

\section{Factors influencing tidal breathing measurements}

The biggest difference between the techniques used in this study was with respect to the apparatus dead space. In contrast to the FTT, the $V \mathrm{D}$, app of the SM 2600 (9 or $18 \mathrm{~mL}$, depending on mask size selected) could have a significant influence on alveolar gas exchange and blood gases. Although the apparatus resistance of the SM 2600 was also higher than that of the FTT $(0.5$ versus $0.2 \mathrm{kPa} \cdot \mathrm{L}^{-1} \cdot \mathrm{s}$ respectively), both are considerably smaller than the infant's own resistance and it is, 
therefore, unlikely that this difference in resistive load between methods made any major contribution to the observed differences in the tidal breathing parameters. As shown in table 1 , body weight was less than $2,800 \mathrm{~g}$ in half of the infants studied, and in such infants the intrinsic resistance is likely to be nearly ten times higher that that of the apparatus, even when using the SM 2600 [25]. While the air stream from the background flow on the infant's face could potentially influence measured tidal breathing parameters when using the FTT, it has been previously shown that this flow has no significant influence on breathing pattern, provided infants are allowed to adapt for a sufficient period of time [26]. All other factors that could potentially influence tidal breathing were the same for both techniques.

Ideally, order of measurements would have been randomised, whereas in this study the FTT was always applied first. This was to ensure that the two sets of measurements could be obtained as closely in time as possible without any interruption of infant's sleep. Had the SM 2600 been applied first in some infants, a much longer interval would have been required for "wash out" because, in contrast to FTT, measurements with the SM 2600 have a significant influence on both tidal breathing parameters [27] and blood gases. Such a prolonged waiting time (>30 min) would have hampered the comparability of the measured parameters and decreased the feasibility of completing the protocol in many of the infants. It has previously been shown that actual duration of measurements may also influence tidal breathing parameters [27]. The FTT allows the investigator to wait until a steady state in the parameters has occurred, whereas during measurements with the SM 2600, the first regular breaths within the first 1-2 min after placement of the face mask have to be used due to the dead space ventilation.

Important differences also exist between the two devices in the estimation of breath-to-breath variability which precludes direct comparisons. For the SM 2600, this is based on user selection of breathing cycles with large amplitudes and minimal disturbances and deformations [28], which has a high risk of subjective bias [5]. In contrast, consecutive evaluation of a sufficient number of breathing cycles was used in the FTT to ensure a more representative evaluation, as described previously [29]. However, since RFTs were only performed in sleeping infants with a regular breathing pattern, this should not have been a source of discrepancy in this particular study.

\section{Differences in the measured tidal breathing parameters}

The increase in $V \mathrm{~T}$ and $f \mathrm{R}$ seen during measurements in infants with the SM 2600 (table 2), are in agreement with a previous study which found increases in the variables of about 25 and $20 \%$ respectively, within $2-3$ min of mask placement when using the same equipment [27]. This was primarily attributed to carbon dioxide $\left(\mathrm{CO}_{2}\right)$ rebreathing due to the high apparatus dead space. The fact that tidal breathing parameters measured with the conventional technique are so dependent on the time of measurement after placement of the face mask, increases the difficulties in attempting to standardize such measurements.

The parameter TEF25 described the end-expiratory course of the TBFVL and was significantly higher when measured with the SM 2600 (table 2). This may reflect the fact that such flows were measured at a higher lung volume due to the increased $V \mathrm{~T}$ per se and/or dynamic elevation of the end expiratory level, secondary to the increased $f \mathrm{R}$ and decreased $t \mathrm{E}$ with this device $[30,31]$. In this study, the greatest differences between measurement techniques were with respect to $t \mathrm{PTEF} / \mathrm{tE}$ and $V \mathrm{PTEF} / V \mathrm{~T}$, with values being reduced by up to $50 \%$ when compared with the FTT. It is unlikely that such differences can simply be attributed to the physiological changes in $V \mathrm{~T}$ and $f \mathrm{R}$ induced by the added dead space, particularly since similar errors can be identified during in vitro simulations [18]. During in vitro validation studies, no differences were observed in the raw flow signal obtained from the two devices, nor between values calculated automatically or manually from the FTT [18]. Similarly, the deviations between the FTT and SM 2600 were $<3 \%$ for $V \mathrm{~T}, f \mathrm{R}$ and $V^{\prime} \mathrm{E}$. However, when compared either with manually calculated values or those derived automatically from the FFT, there was a significant underestimation of shape-dependent parameters such $t$ PTEF/ $t$ E derived by the SM 2600, with errors being up to $60 \%$ when flows simulating those observed in preterm neonates were applied. These errors appear to result from differences in signal processing and can only be detected if appropriate nonsinusoidal flow profiles, representing those seen in infants, are used to evaluate equipment [18].

Both $t \mathrm{PTEF} / t \mathrm{E}$ and $V \mathrm{PTEF} / V \mathrm{~T}$ are not only critically dependent on the accurate determination of PTEF, but on the accurate determination of zero flow and hence, start of expiration. In the FTT, which was developed recently, considerable efforts were made to ensure correct breath detection even in the smallest infants, by using a flow-threshold algorithm with an additional plausibility check for the zero crossings that were detected [32]. Further validation was performed by comparing results that were derived automatically with manual calculations of the time-based trace and TBFVL. Unfortunately, such an approach was not feasible with the SM 2600, since this was developed over 15 yrs ago, before recent technological advances in equipment and software. Since the algorithms used by the SM 2600 for signal evaluation were not available, and manual evaluation of the graphical presentation was not feasible, detailed analysis of the source of the discrepancies was not possible. The results from this study suggest that equipment for infant RFTs needs to be carefully checked using a suitable in vitro model before clinical application [18, $33,34]$. Sinusoidal model pumps, which are commonly used to investigate the accuracy of devices for infant RFT, are not necessarily appropriate. Instead, model pumps with adjustable flow profiles covering the relevant measuring range, should be used in such assessments [18].

\section{Clinical implications}

It is a fundamental principle of all physiological 
measurements that measuring procedures should have no or minimal influence on the parameters being measured, and that different techniques and equipment should lead to the same results. However, this demand is extremely difficult to achieve when measuring tidal breathing in newborns. The results from this study suggest that tidal breathing parameters measured with the FTT differ significantly from those collected with conventional systems that have an appreciable dead space. Hopefully, in the near future, precise flowmeters with negligible flow resistance (e.g. anemometers [35] or ultrasonic sensors [36]) will become routinely available, so that, in addition to the elimination of apparatus dead space by the FTT, the resistive load can also minimized.

One important message from this study is that some differences (especially in shape-dependent parameters like time to peak tidal expiratory flow as a proportion of tidal expiratory time) are so large that there is currently no comparability in the measured tidal breathing parameters collected using different devices, and diagnostic misinterpretations are, therefore, likely. The fact that tidal breathing parameters in newborns are currently so method-dependent makes it difficult to compare data between different laboratories and impossible to pool data for a meta-analysis (e.g. for establishment of reference ranges). Furthermore, reference values which have been determined with any one particular system [37] are unlikely to be of relevance when using other measurement techniques or equipment. Thus, there is an urgent need to standardize the technical requirements for respiratory function testing in newborns, with respect to both equipment and software.

\footnotetext{
Acknowledgements. The authors thank M. Schmidt for support in the development of the software used and D. Windstetter and S. Schmidt for their assistance in respiratory function testing.
}

\section{References}

1. Stocks J, Sly PD, Tepper RS, Morgan WL. Infant respiratory function testing. New York, Wiley-Liss, 1996.

2. Stick SM, Ellis E, LeSouef PN, Sly PD. Validation of respiratory inductance plethysmography ("Respitrace") for the measurement of tidal breathing parameters in newborns. Pediatr Pulmonol 1992; 14: 187-191.

3. Edberg KE, Sandberg K, Silberberg A, Ekstrom Jodal $B$, Hjalmarson $\mathrm{O}$. Lung volume, gas mixing, and mechanics of breathing in mechanically ventilated very low birth weight infants with idiopathic respiratory distress syndrome. Pediatr Res 1991; 30: 496-500.

4. Brown K, Aun C, Jackson E, Meckersie A, Hatch D, Stocks J. Validation of respiratory inductive plethysmography using the Qualitative Diagnostic Calibration method in anaesthetized infants. Eur Respir $J$ 1998; 12: 935-943.

5. Ahlström H, Jonson B. Pulmonary mechanics in infants. Methodological aspects. Scand J Respir Dis 1974; 55: 129-140.
6. Fleming PJ, Levine MR, Goncalves A. Changes in respiratory pattern resulting from the use of a face mask to record respiration in newborn infants. Pediatr Res 1982; 16: 1031-1034.

7. Dolfin T, Duffty P, Wilkes D, England S, Bryan H. Effects of a face mask and pneumotachograph on breathing in sleeping infants. Am Rev Respir Dis 1983; 128: 977-979.

8. Marsh MJ, Ingram D, Milner AD. The effect of instrumental dead space on measurement of breathing pattern and pulmonary mechanics in the newborn. Pediatr Pulmonol 1993; 16: 316-322.

9. Schmalisch G, Wauer RR. Current technical limitations of lung function testing in ventilated infants and infants breathing spontaneously. Paediatr Grenzgeb 1995; 34: 21-37.

10. Morris MG. A simple new technique to measure the effective dead space of the face mask with a water volumeter in infants. Eur Respir J 1999; 14: 1163-1166.

11. Ruttimann UE, Galioto FM J, Franke JR, Rivera O. Measurement of tracheal airflow in newborns by a differential flow system. Crit Care Med 1981; 9: 801804.

12. Thomson A, Elliott J, Silverman M. Pulmonary compliance in sick low birthweight infants. How reliable is the measurement of oesophageal pressure? Arch Dis Child 1983; 58: 891-896.

13. Schmalisch G, Wauer RR. Adjustment of the background flow for measurement of ventilation in newborn infants using the flow-through technique. Pneumologie 1995; 49: 461-465.

14. Wauer RR, Maurer T, Nowotny T, Schmalisch G. Assessment of functional residual capacity using nitrogen washout and plethysmographic techniques in infants with and without bronchopulmonary dysplasia. Int Care Med 1998; 24: 469-475.

15. Prechtl HF. The behavioural states of the newborn infant (a review). Brain Res 1974; 76: 185-212.

16. Lodrup KC, Mowinckel P, Carlsen KH. Lung function measurements in awake compared to sleeping newborn infants. Pediatr Pulmonol 1992; 12: 99-104.

17. Sly PD, Davis GM. Equipment requirements for infant respiratory function testing. In: Stocks J, Sly PD, Tepper RS, Morgan WJ, eds. Infant respiratory function testing. New York, Wiley-Liss; 1996; pp. 4580 .

18. Schmalisch G, Foitzik B, Wauer RR, Stocks J. In vitro assessment of equipment and software to assess tidal breathing parameters in infants. Eur Respir J 2001; 17: $100-107$.

19. Foitzik B, Schmidt M, Windstetter D, Wauer RR, Schmalisch G. Leak measurements in spontaneously breathing premature newborns by using the flowthrough technique. J Appl Physiol 1998; 85: 11871193.

20. Bland JM, Altman DG. Statistical methods for assessing agreement between two methods of clinical measurement. Lancet 1986; 1: 307-310.

21. Turner MJ, MacLeod IM, Rothberg AD. Effects of temperature and composition on the viscosity of respiratory gases. J Appl Physiol 1989; 67: 472-477.

22. Andreasson B, Lindroth M, Svenningsen NW, Jonson B. Effects on respiration of CPAP immediately after extubation in the very preterm infant. Pediatr Pulmonol 1988; 4: 213-218.

23. Schmalisch G, Wauer RR, Böhme B. Changes in 
pulmonary function in preterm infants recovering from RDS following early treatment with Ambroxol: Results of a randomized trial. Pediatr Pulmonol 1999; 27: 104-112.

24. Schmidt M, Foitzik B, Wauer RR, Hochmuth O, Schmalisch G. In vitro investigations of jet-pulses for the measurement of respiratory impedance in newborns. Eur Respir J 1999; 14: 1156-1162.

25. Gappa M, Rabbette PS, Costeloe KL, Stocks J. Assessment of passive respiratory compliance in healthy preterm infants: a critical evaluation. Pediatr Pulmonol 1993; 15: 304-311.

26. Windstetter D, Foitzik B, Schmalisch G, Wauer RR. Long term tidal breathing measurements in newborns: influence of a bias flow on ventilatory parameters. Eur J Med Res 1998; 4: 15-22.

27. Emralino F, Steele AM. Effects of technique and analytic conditions on tidal breathing flow volume loops in term neonates. Pediatr Pulmonol 1997; 24: 86-92.

28. Lodrup-Carlsen KC. Tidal breathing analysis in infants and preschool children: tidal flow-volume loops. Eur Respir Mon 1997; 5: 27-57.

29. Stocks J, Dezateux CA, Jackson EA, Hoo AF, Costeloe KL, Wade AM. Analysis of tidal breathing parameters in infancy: how variable is $t \mathrm{PTEF} / \mathrm{tE}$. $A m$ J Respir Crit Care Med 1994; 150: 1347-1354.

30. Kosch PC, Stark AR. Dynamic maintenance of end- expiratory lung volume in full-term infants. $J$ Appl Physiol 1984; 57: 1126-1133.

31. Stark AR, Cohlan BA, Waggener TB, Frantz ID, Kosch PC. Regulation of end-expiratory lung volume during sleep in premature infants. J Appl Physiol 1987; 62: 1117-1123.

32. Schmidt M, Foitzik B, Wauer RR, Winkler F, Schmalisch G. Comparative investigations of algorithms for the detection of breaths in newborns with disturbed respiratory signals. Comput Biomed Res 1998; 31: 413-425.

33. Frey U, Stocks J, Coates A, Sly P, Bates J. Specifications for equipment used for infant pulmonary function testing. Eur Respir J 2000; 16: 731-740.

34. Frey U, Stocks J, Sly P, Bates J. Specifications for signal processing and data handling used for infant pulmonary function testing. Eur Respir $J$ 2000; 16: 1016-1022.

35. Plakk P, Liik P, Kingisepp PH. Hot-wire anemometer for spirography. Med Biol Eng Comput 1998; 36: 1721.

36. Buess C, Pietsch P, Guggenbuhl W, Koller EA. A pulsed diagonal-beam ultrasonic airflow meter. $J$ Appl Physiol 1986; 61: 1195-1199.

37. Lodrup-Carlsen KC, Magnus P, Carlsen KH. Lung function by tidal breathing in awake healthy newborn infants. Eur Respir J 1994; 7: 1660-1668. 